\title{
A pilot study on the melatonin treatment in patients with early septic shock: results of a single-center randomized controlled trial
}

\author{
Abbas Taher $^{1} \cdot$ Farnaz Shokoohmand $^{2} \cdot$ Elham Abdoli $^{3} \cdot$ Younes Mohammadi ${ }^{4}$ Maryam Mehrpooya ${ }^{2}(\mathbb{D}$
}

Received: 8 June 2021 / Accepted: 25 August 2021 / Published online: 1 September 2021

(c) Royal Academy of Medicine in Ireland 2021

\begin{abstract}
Background We assessed the potential impact of a high dose of melatonin treatment in patients with early septic shock. Methods Forty patients with early septic shock were randomly allocated to the melatonin or placebo groups. Besides standard-of-care treatment, melatonin and placebo were administered at a dose of $50 \mathrm{mg}$ for five consecutive nights. The efficacy outcomes were severity of organ dysfunction based on the Sequential Organ Failure Assessment (SOFA) score, the number of patients requiring mechanical ventilation and ventilator-free days, the mean required vasopressor dose and vasopressor-free days, and 28 days all-cause mortality.

Results After 5-day treatment, the mean SOFA scores decreased $4.05 \pm 4.75$ score in the melatonin group and $2.25 \pm 4.87$ in the placebo group. On day $28,60 \%$ of the melatonin-treated patients and $35 \%$ of the placebo-treated patients had a SOFA score below six. Thirteen cases in the placebo group and nine cases in the melatonin group required mechanical ventilation; however, there was no statistically significant difference between the groups regarding these outcomes. The melatonintreated patients had more ventilator-free days than placebo-treated patients over the 28 -day $(16.90 \pm 9.24$ vs. $10.00 \pm 10.94$; $p$ value $=0.035$ ). The mean reduction in the required dose of vasopressor was $6.2 \pm 5.12$ in the melatonin-treated patients compared to $3.20 \pm 3.95$ in the placebo-treated patients $(p$ value $=0.045)$. Vasopressor-free days in the melatonin-treated group were also significantly more than the placebo-treated group $(12.75 \pm 7.43$ days vs. $10.15 \pm 6.12$ days; $p$ value $=0.046)$. Conclusions Our pilot study supported the potential benefits of melatonin in treating septic shock. Further clinical evidence is required for expanding and confirming these findings.
\end{abstract}

Trial registration The trial was registered at Clinicaltrials.gov (ID code: IRCT20120215009014N296). Registration date: 15/09/2019.

Keywords Inflammation $\cdot$ Melatonin $\cdot$ Oxidative stress $\cdot$ Septic shock

\section{Background}

Sepsis is a clinical syndrome occurring when the host immune response to an infectious insult spreads beyond the site of infection, leading to a more generalized response.

Maryam Mehrpooya

m_mehrpooya2003@yahoo.com; m.mehrpoya@umsha.ac.ir

1 Department of Anesthesiology and Critical Care, Hamadan University of Medical Sciences, Hamadan, Iran

2 Department of Clinical Pharmacy, School of Pharmacy, Hamadan University of Medical Sciences, Hamadan, Iran

3 Department of Infectious Disease, School of Medicine, Hamadan University of Medical Sciences, Hamadan, Iran

4 Modeling of Noncommunicable Diseases Research Center, School of Public Health, Hamadan University of Medical Sciences, Hamadan, Iran
Its severity ranges from infection and bacteremia to severe sepsis and septic shock. Septic shock is produced by the sepsis presence with refractory hypotension. It is defined as systolic blood pressure (SBP) below $90 \mathrm{mmHg}$ or a reduction in SBP of $>40 \mathrm{mmHg}$ in adults, despite adequate fluid resuscitation [1]. Although the strategies with a focus on timely antibiotic administration and aggressive resuscitation are extensively implemented for their treatment, severe sepsis and septic shock are still associated with unacceptably high rates of mortality [2]. Although the precise mechanism of sepsis is not still understood, its cause is likely multifactorial. It involves the direct impact of invading microorganisms or their toxic products, the excessive releasing quantities of proinflammatory mediators, activation of the complement system, and coagulation mechanisms that ultimately lead to widespread cellular injury. With septic shock, insufficient oxygen relative to oxygen needs leads to cellular ischemia 
and hypoxia. Failure of cellular perfusion causes a noticeable rise in reactive oxygen species (ROS) and a reduction in endogenous antioxidant defense, which increases the already high state of oxidative stress [3]. Further, as is amply demonstrated, oxidative stress and the inflammatory response are closely related and one of which can be easily induced by another [4]; as a result, under the pathogenic inflammatory condition of septic shock, ROS production is increased, and antioxidant protection reduced. In fact, with septic shock, inflammation together with the hypoxia induces a state of oxidative stress, a decrease in antioxidant activity, and a loss of regulatory mechanisms that ultimately leads to mitochondrial dysfunction and failure of energy production [5]. Thus, during septic shock, in addition to the defect in delivery of $\mathrm{O}_{2}$ in the blood vessels and capillaries, due to the sepsis-induced mitochondrial dysfunction, impaired $\mathrm{O}_{2}$ utilization is occurred, which reduces cellular energy production. Consequently, mitochondrion becomes an origin of the generation of excessive ROS in sepsis, which further increases the damage to mitochondria. The resulting wide mitochondrial damage and insufficient energy production can trigger organ dysfunction and death in affected patients [6]. All of the abovementioned statements point to the fact that the therapeutic strategy targeting overwhelming inflammatory responses and a high state of oxidative stress and maintaining the proper functioning of mitochondria is critical in sepsis management [7].

Melatonin is a hormone primarily synthesized and secreted from the pineal gland that the nocturnal rise in its secretion has an essential role in the regulation of the sleep-wake cycle, pubertal development, and seasonal adaptation [8]. There is growing evidence on the other beneficial effects of melatonin, including locomotor activityregulating, antioxidant, anti-inflammatory, antinociceptive, pain-modulating, antidepressant, anxiolytic, antineophobic, vascular, retinal, neuroprotective, blood pressure-regulating, and anti-tumor effects [9-11]. Because of this numerous functional melatonin properties, in the last decade, both preventive and medical use of melatonin for a number of clinical states has attracted much attention [12]. Considering its unique immunoregulatory functions, together with potent anti-inflammatory, antioxidant, and free radical scavenger properties, melatonin has received progressively scientific attention in the management of various pathophysiological conditions in which high state oxidative stress and inflammation are engaged in their pathophysiologic processes [13]. In this vein, melatonin has been proposed as a potential therapeutic candidate for the treatment and management of sepsis [14]. It is speculated that the potential benefits of melatonin in the treatment of sepsis may also stem from its ability to optimize and restore mitochondrial function [15]. On the other hand, from a pathological viewpoint, there is growing evidence regarding the possible disturbed circadian rhythm of melatonin release in patients with severe sepsis [16-18]. Further, encouraging data obtained from a number of studies in animal models of septic shock showed that melatonin supplementation might exert protective effects against multiorgan failure in septic settings [19-22]. Based on this evidence, we hypothesized that adult patients with septic shock might be likely to benefit from exogenous melatonin administration. Thus, we designed a randomized, doubleblind, placebo-controlled pilot trial to evaluate whether a high dose of melatonin (50 $\mathrm{mg}$ for 5 days) administration as adjunctive therapy to standard therapeutic care can be beneficial in improving the clinical status of patients with the diagnosis of early septic shock.

\section{Material and methods}

\section{Study design}

This study was a prospective, two-arm, double-blind, randomized clinical trial in adult patients with early septic shock. It was carried out at a single tertiary care referral hospital in West of Iran, with combined adults Medical and Surgical Intensive Care Unit (ICU) of 30 beds (20-bed surgical ICU and 10-bed medical ICU). The trial was performed in accordance with the Declaration of Helsinki and the International Good Clinical Practice guides, and the registered research protocol was approved by the local Ethics Committee of Hamadan University of Medical Sciences (approval number: IR.UMSHA.REC.1398.341) before initiation. The written informed consent was provided by the patients or their first-degree relatives if they were unable to provide consent. The trial was registered at the Iranian Registry of Clinical Trials (www.irct.ir) on 15/09/2019, with registration number "IRCT20120215009014N296." The investigators, ICU nurses, physicians, and patients were blinded to the treatment allocations. The study drug (melatonin or placebo) was prepared, hooded, and dispensed by a hospital pharmacist. Patient enrollment was between September 2019 and March 2021. After data collection and analyses, treatment allocation was first revealed to the study researchers.

\section{Patient enrollment}

The sample size was calculated as 20 in each group based on Zabet et al. [23] to detect a difference of at least six or more in the required vasopressor dose between the two groups with a standard deviation (SD) of six, while considering $\alpha=0.05,20 \%$ drop-out, and a power of $80 \%$. During the study period, all adult critically ill patients, between 18 and 75 years old, with a diagnosis of septic shock, ongoing treatment with vasopressors for at least $6 \mathrm{~h}$ and less than $24 \mathrm{~h}$ who had a central venous catheter were eligible for inclusion 
in the trial. The definition of the septic shock was as follows: the presence of documented or suspected infection, at least two organ failure, each defined by an organ-specific Sequential Organ Failure Assessment (SOFA) [24] score of 3 or 4, and persisting hypotension requiring vasopressors for maintaining the mean blood pressure of at least $65 \mathrm{~mm} \mathrm{Hg}$ or SBP of at least $90 \mathrm{~mm} \mathrm{Hg}$ in the absence of hypovolemia [2]. Patients younger than 18 years; pregnant and lactating patients; currently on melatonin supplementation; having a history of allergy or intolerance to melatonin; unable to receive enteral medication; having cardiogenic shock; diagnosis with late septic shock at presentation with multiple organ failure; having underlying clinical conditions that could impact short-term survival, including malignancies, patients under immunosuppressive therapy, having a preexisting chronic kidney disease and chronic liver disease; having uncontrolled hemorrhage; and moribund patient not expected to survive $24 \mathrm{~h}$ were excluded from the trial. Further, patients who died within $24 \mathrm{~h}$ of enrolment were also dropped out from the trial.

\section{Intervention}

Forty patients who fulfilled inclusion and exclusion criteria in a 1:1 ratio by block randomization method (in four blocks) were allocated to the melatonin or placebo groups. The randomization was performed by a researcher not engaged in managing patients. The trial medications were started immediately after randomization. Melatonin at a dosage of $50 \mathrm{mg}$ (Razak Pharmaceutical Company, Tehran, Iran) was administrated as a liquid oral solution at 9 p.m. for five consecutive nights. Placebo oral solution was similarly prepared and was indiscernible from active treatments. The trial medications were stopped without tapering off at the end of the intervention period. The solutions of the study medications were made ready by a hospital pharmacist that was not masked to treatment and was not a further part of those patients' care. The medication was administrated through the nasogastric tube in patients with a disorder of consciousness or swallowing deficit (dysphagia).

The protocol-based care was provided for all patients irrespective of the treatment group according to the 2016 Surviving Sepsis Campaign recommendations [25]. Those included placements of a central venous catheter for monitoring central venous oxygen saturation $(\mathrm{ScvO} 2)$ and pressure and for administering intravenous fluids, intravenous broad-spectrum antibiotics as soon as possible following taking specimens from the possible infection sites, monitoring of blood glucose levels at least and maintained blood glucose at $\leq 150 \mathrm{mg} / \mathrm{dl}$ by infusing insulin intravenously, intravenous replacement fluids, vasopressor therapy, transfusion of blood products, nutrition support, renal replacement therapy, prophylactic anticoagulation, and prevention of stress ulcer according to the international guidelines for severe sepsis. Daily monitoring is similar to the routine monitoring of these patients as part of their ICU management.

Further, throughout the active treatment period, an ICU man, who was not affiliated with the study, independently visited patients daily to identify possible clinical adverse events. He assessed any alteration in physical signs and symptoms and laboratory parameters of the study patients and determined if the alteration was clinically important and different from what was expected in the course of the treatment of septic shock patients. In case of any occurrence of clinically significant and unexpected adverse experience that was thought to be associated with the study drug, they were recorded as adverse events.

\section{Outcomes}

At enrollment, the following data were recorded for each study patient: (1) demographic characteristics; (2) time of admission in ICU and hospital; (3) time from septic shock onset; (4) coexisting illnesses; (5) concomitant medications; (6) hemodynamic parameters and laboratory tests; (7) cultures and Gram examination from the suspected infection sites; (8) type and dose of any antibiotics; (9) type and dose of inotropes and vasopressors; (10) Acute Physiology and Chronic Health Evaluation (APACHE) disability scale at the first $24 \mathrm{~h}$ of ICU admission; and (11) illness severity based on SOFA score.

The SOFA score was used for determining the organ dysfunction severity associated with sepsis in the study patients. The SOFA score as a simple and objective measurement tool has been widely used for quantifying the degree of organ dysfunctions in critically ill patients. SOFA is on the basis of six different scores for each organ system (cardiovascular, respiratory, hepatic, neurologic, renal, and coagulation systems). The score of each system is ranged from 0 to 4 , and the sum of these component scores represents the total maximum SOFA scores ( $0-24$ points). The highest and mean scores are most predictive of mortality. The presence of the organ system failure for each of these six organ systems is defined as a SOFA score $\geq 3$ [24].

The primary efficacy endpoints were as follows: 1 , the change in SOFA scores at 24, 48, 72, and $96 \mathrm{~h}$ after treatment compared to the start of treatment and the percentage of patients who recovered from organ failure defined as SOFA score below 6 at day 28; 2, the number of patients who required invasive mechanical ventilation and ventilatorfree days up to day 28; and 3, mean vasopressor dose at 24, 48,72 , and $96 \mathrm{~h}$ from enrolment and vasopressor free days up to day 28. As the secondary endpoints, the following clinical outcomes were compared in the study groups: 1 , 28 days all-cause mortality; 2 , the percentage of patients who had acute renal failure and needed renal replacement 
therapy up to day 28; and 3, the length of ICU and hospital stay (up to day 60).

\section{Statistical analyses}

IBM, SPSS version 20 (SPSS Inc., Chicago, IL, USA) was used for statistical analysis. Data analysis was performed for the intent-to-treat (ITT) population, and the last observation carried forward (LOCF) method was employed for missing data handling. Shapiro-Wilk test was used for examining the normal distribution of continuous data. The normally and non-normally, continuous data were expressed as mean (standard deviation (SD)) and median (interquartile range (IQR)), respectively, while categorical data were expressed as numbers with percentages. Independent $t$ test and Mann-Whitney $U$ test were used to compare the mean $( \pm \mathrm{SD})$ and median $( \pm \mathrm{IQR})$ of continuous variables between the drug and placebo group, respectively. The chi-square test was used to compare the proportions/test the association between groups. For repeated observations over time, Friedman's analysis of variance (ANOVA) was used to estimate the significance level among the time points. If, in Friedman's ANOVA, the $p$ value was observed to be significant, then the difference in medians between individual groups was further assessed using the Wilcoxon signed-rank test. $p$ value $\mathrm{s}<0.05$ were considered statistically significant.

\section{Results}

\section{Demographics and baseline characteristics}

The flowchart of trial enrollment is as depicted in Fig. 1. Between September 2019 and March 2021, eighty-eight cases were evaluated for eligibility, of which 48 cases were excluded (43 cases did not fulfill inclusion criteria, and 5 cases refused to participate). Of the 40 patients fulfilling the inclusion/exclusion criteria and given written informed consent, 20 cases were allocated to the intervention group, and 20 cases were allocated to the control group and were followed for 28 days (Fig. 1). All participants received at least one dose of the trial medication; so, after randomization, there were not any patients excluded from the analysis after randomization, and all randomly allocated cases were included in the final intention-to-treat analysis. Nineteen cases (95\%) in the melatonin group and eighteen cases (90\%) in the placebo group received the entire 5-day treatment course. Table 1 presents the demographic and clinical features of the participants at enrollment. The patients' average age was $54.9 \pm 12.21$ years, and $67.5 \%$ of the patients were men. As shown at the start of the trial, the groups were well balanced in terms of baseline clinical features, including general condition, coexisting illnesses, the focus of sepsis,

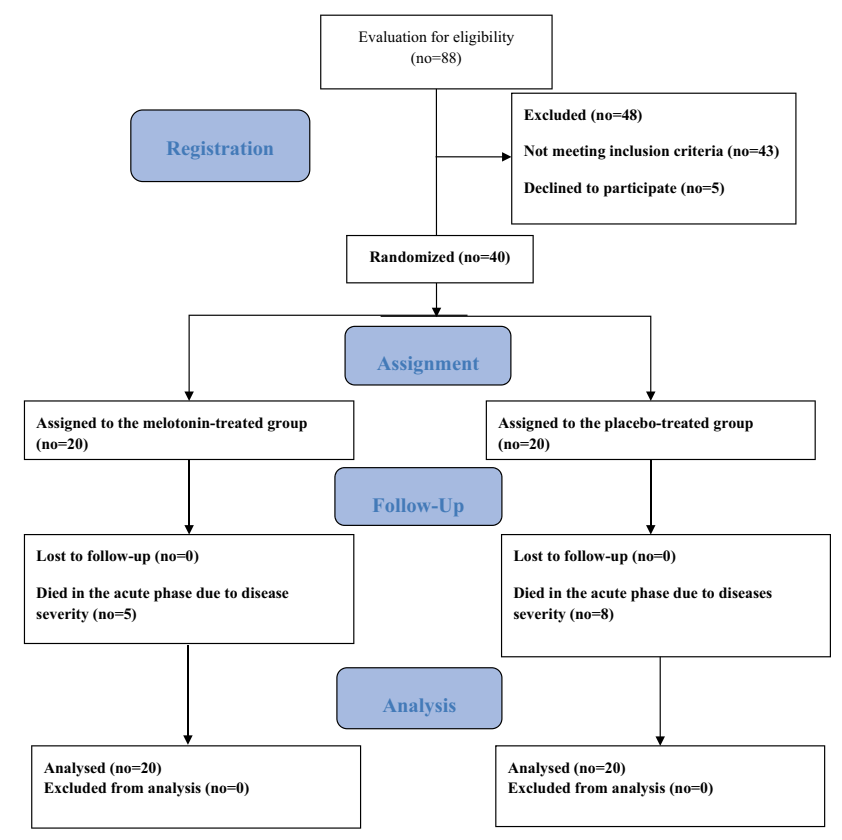

Fig. 1 The study flow diagram

vital signs, and laboratory tests. In both groups, the respiratory tract was the most common site of infection, followed by intra-abdominal infections. Regarding the illness severity scores at enrollment, the SOFA and APACHE II scores were also comparable in the two groups ( $p$ value $=0.74$ and $p$ value $=0.64$, respectively). Interventions provided for the septic shock management at study enrollment were similar in both groups. In all study patients, the initial antimicrobial treatment based on the infection site and the pathogen sensitivity was judged adequate. The adjunctive steroid was administrated in six cases in the treatment group (30\%) and eight cases in the placebo group (40\%) that was comparable in the two groups $(p$ value $=0.74)$.

\section{Efficacy outcomes}

Figure 2 and Table 2 depict the results related to the melatonin impact on the primary and secondary clinical efficacy outcomes. Five of 20 cases (25\%) in the melatonin group and 8 of 20 cases (40\%) in the control group had died within the 28-day follow-up period (Table 2). Despite the lower mortality rate in the melatonin recipients compared to the placebo recipients during the 28-day follow-up, no statistically significant difference was observed between the study groups $(p$ value $=0.51)$. According to the general linear model analysis of data, although through the 5-day intervention period, more improvement in the organ dysfunction severity assessed by SOFA score was noted in the patients treated with melatonin in comparison with the patients treated with placebo, it was not statistically significant $(p$ 
Table 1 Baseline demographics and clinical features of the intention-to-treat population

\begin{tabular}{|c|c|c|c|}
\hline Variable & $\begin{array}{l}\text { Melatonin group ( } 20 \\
\text { patients) }\end{array}$ & $\begin{array}{l}\text { Placebo group }(20 \\
\text { patients) }\end{array}$ & $p$ value \\
\hline \multicolumn{4}{|l|}{ Characteristic } \\
\hline Age, years, mean $\pm S D$ & $55.75 \pm 11.45$ & $53.95 \pm 13.17$ & 0.65 \\
\hline $\operatorname{Sex}(M / F)$, no. $(\%)$ & $13 / 7(65 / 35)$ & $14 / 6(70 / 30)$ & 1.00 \\
\hline $\mathrm{BMI}$, mean $\pm \mathrm{SD}$ & $25.50 \pm 3.40$ & $24.90 \pm 3.10$ & 0.64 \\
\hline \multicolumn{4}{|l|}{ Coexisting illnesses } \\
\hline Cardiovascular diseases, no. (\%) & $10(50.0)$ & $11(55.0)$ & 1.00 \\
\hline Diabetes, no. (\%) & $9(45.0)$ & $6(30.0)$ & 0.51 \\
\hline Respiratory diseases, no. (\%) & $7(35.0)$ & $5(25.0)$ & 0.73 \\
\hline Chronic renal failure, no. (\%) & $2(10.0)$ & $3(15.0)$ & 1.00 \\
\hline Neurologic disorders, no. (\%) & $5(25.0)$ & $4(20.0)$ & 1.00 \\
\hline Other, no. $(\%)$ & $6(30.0)$ & $4(20.0)$ & 0.72 \\
\hline \multicolumn{4}{|l|}{ Source of infection } \\
\hline Respiratory infection, no. (\%) & $8(40.0)$ & $8(40.0)$ & \\
\hline Intraabdominal infection, no. (\%) & $4(20.0)$ & $5(25.0)$ & 0.88 \\
\hline Urinary tract infection, no. (\%) & $4(20.0)$ & $2(20.0)$ & \\
\hline Others or un-known infection, no. (\%) & $4(20.0)$ & $5(25.0)$ & \\
\hline Blood culture positive, no. (\%) & $7(35.0)$ & $6(30.0)$ & 1.00 \\
\hline \multicolumn{4}{|l|}{$\begin{array}{l}\text { Vital sign and laboratory data at enrollment, } \\
\text { mean } \pm \text { SD }\end{array}$} \\
\hline Temperature, ${ }^{\circ} \mathrm{C}$ & $37.8( \pm 0.8)$ & $37.9( \pm 0.9)$ & 0.73 \\
\hline Respiratory rate, times/min & $21 \pm 2$ & $20 \pm 3$ & 0.58 \\
\hline Heart rate, times/min & $97 \pm 16$ & $95 \pm 18$ & 0.78 \\
\hline Systolic blood pressure (mmHg) & $102.8 \pm 9.3$ & $104.5 \pm 9.7$ & 0.59 \\
\hline Mean arterial pressure $(\mathrm{mmHg})$ & $76.3 \pm 11.4$ & $78.2 \pm 12.1$ & 0.60 \\
\hline White blood cell count $\left(10^{3} / \mathrm{L}\right)$ & $10.6 \pm 6.5$ & $9.7 \pm 7.2$ & 0.69 \\
\hline Platelet count $\left(10^{3} / \mathrm{L}\right)$ & $151 \pm 96$ & $144 \pm 94$ & 0.81 \\
\hline Creatinine (mg/dL) & $1.7 \pm 0.6$ & $1.8 \pm 0.5$ & 0.71 \\
\hline Total bilirubin (mg/dL) & $1.4 \pm 0.7$ & $1.3 \pm 0.5$ & 0.62 \\
\hline \multicolumn{4}{|l|}{ Severity of illness at enrollment } \\
\hline APACHE-II, mean \pm SD & $22 \pm 6$ & $21 \pm 7$ & 0.64 \\
\hline $\mathrm{SOFA}$, mean $\pm \mathrm{SD}$ & $12.30 \pm 3.50$ & $11.90 \pm 3.10$ & 0.74 \\
\hline
\end{tabular}

$B M I$ body mass index, $S D$ standard deviation, IQR interquartile range, APACHE II Acute Physiology and Chronic Health Evaluation II, SOFA Sequential Organ Failure Assessment value $=0.29$; Fig. 2a). The mean SOFA score decreased from $12.30 \pm 3.50$ at the time of enrollment to $8.20 \pm 5.68$ at the fifth day of treatment in the melatonin group and decreased from $11.90 \pm 3.10$ to $9.75 \pm 5.75$ in the placebo group. The proportion of patients who recovered from organ failure defined as SOFA score below six at day 28 was $60 \%$ (12 of 20 patients) in the melatonin-treated patients versus $35 \%$ ( 7 of 20 patients) in the placebo-treated patients (Table 2); however, there was no statistically significant difference between the study groups ( $p$ value $=0.21$ ).

During 28 days of follow-up, nine cases in the intervention group (45\%) and thirteen cases in the control group $(65 \%)$ required invasive ventilatory support that was not a significant difference ( $p$ value $=0.33$ ). By day 28 , in comparison with the patients treated with placebo, the patients treated with melatonin had significantly more ventilator-free days $(16.90 \pm 9.24$ days in the melatonin group vs. $10.00 \pm 10.94$ days in the placebo group; $p$ value $=0.035$ ) . The comparison of the number of patients who needed renal replacement therapy to day 28 in the melatonin group did not show a significant differences from the placebo group (melatonin group $=3$ patients [15\%], placebo group $=5$ patients $[25 \%] ; p$ value $=0.69$ ).

Figure $2 \mathrm{~b}$ shows the changes in the mean dose of vasopressor through the 5-day intervention period across patient groups. Although compared to placebo-treated patients, the melatonin-treated patients exhibited a significantly higher decline in the mean required dose of the vasopressor through the active treatment period, this trend did not reach statistical significance ( $p$ value $=0.22$ ); however, as a supplemental analysis, a comparison was made between two study groups for the mean change in the required dose of vasopressor at 
a: SOFA score
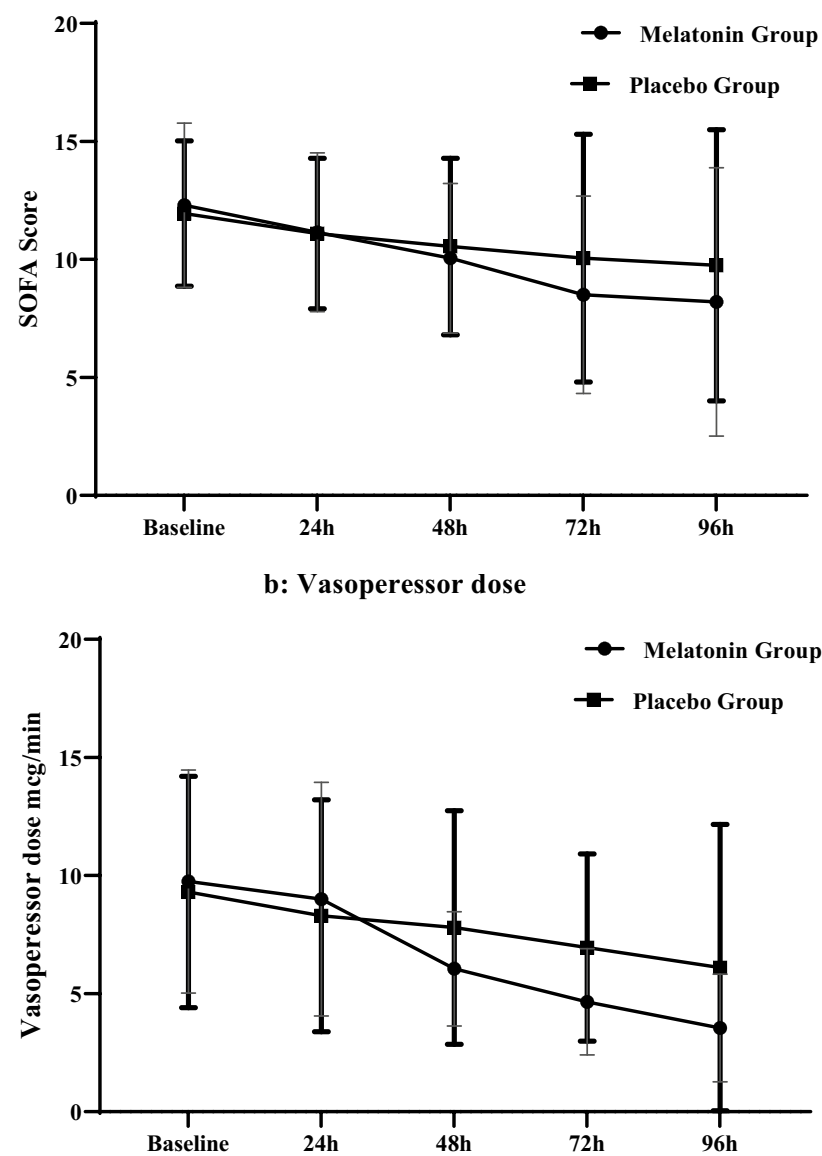

Fig. 2 a Change in the mean SOFA score (Sequential Organ Failure Assessment) for severity of organ dysfunction associated with sepsis through the 5-day intervention period across patient groups ( $p$ value $=0.29)$. b Change in the mean vasopressor dose at different time points during treatment period $(p$ value $=0.22)$

$96 \mathrm{~h}$ compared to baseline. The mean required dose of vasopressor at day 5 of treatment showed a $6.21 \pm 5.12$ reduction in the patients treated with melatonin versus a $3.20 \pm 3.95$ reduction in the patients treated with placebo that was a significant difference ( $p$ value $=0.045$; Table 2 ). Further, through the 28-day follow-up, vasopressor-free days were also significantly higher in the melatonin group compared to the placebo group ( $12.75 \pm 7.43$ days vs. $10.15 \pm 6.12$ days; $p$ value $=0.046$; Table 2). Besides, there were also significant differences between the study groups in terms of the length of hospitalization and ICU stay. The mean day of ICU stay was $15.85 \pm 5.03$ days in the melatonin-treated patients and $21.10 \pm 10.12$ days in the placebo-treated patients, which was a statistically significant difference ( $p$ value $=0.045$ ). Also, a significant difference was observed between the study groups in terms of the mean day of hospitalization ( $18.05 \pm 5.14$ days in the melatonin group vs. $25.85 \pm 7.13$ days in the placebo group; $p$ value $=0.03$ )

\section{Safety outcomes}

As regards possible adverse effects of melatonin, no apparent adverse events occurred during the study period that could be related to the melatonin treatment. Further, during the 5-day active treatment period, no patients were withdrawn due to identified adverse effects or toxicities of melatonin.

\section{Discussion}

The data of this pilot study revealed that compared to placebo, administration a high dose of melatonin $(50 \mathrm{mg}$ for five days) at the septic shock onset as an adjuvant to standards of care modalities could attenuate systemic organ dysfunction associated with sepsis and reduce the demand for mechanical ventilation, although these differences were statistically insignificant. Compared to placebo, melatonin therapy significantly reduced the mean required dose of vasopressor, shortened days on vasopressor treatment and shortened the duration of mechanical ventilation through a 28-day follow-up. Melatonin also decreased the ICU and hospital stay length without any detectable drug-related adverse events or toxicities. Despite these promising outcomes, since the study's sample size was small, there was no statistically significant difference between the intervention and control groups regarding the rate of 28-day all-cause mortality.

Although its pathophysiology is still incompletely understood, it is now well clear that oxidative stress and mitochondria malfunctioning as a result of the over-inflammatory responses to infection are involved in the development of cell and organ injury in sepsis [3]. Indeed, oxidative stress in inflammatory-associated pathogenic conditions such as sepsis causing significant mitochondrial injury. Considering mitochondria's pivotal role in cellular energy production, oxidative stress-mediated damage to mitochondria leads to diminished energy generation, and as a consequence, a further rise in the production of ROS in the mitochondrial matrix. This ROS overload contributes to further mitochondrial injury with the final consequence of exacerbation of oxidative stress and inflammatory process that provokes tissue damage in a vicious cycle [26]. Thus, it has been hypothesized that therapeutic approaches directed to enhance endogenous antioxidant defenses, optimize the mitochondrial function, and modulate the amplitude of inflammatory responses might improve the overall outcome in patients with septic shock [27-29]. In this vein, recently, the potential efficacy of a number of agents targeting these pathophysiologic processes has been investigated in basic research and clinical studies that some of these studies provided promising and favorable results [30-33].

In view of its potent antioxidant and anti-inflammatory properties, in particular, at high doses, exogenously 
Table 2 Primary and secondary clinical outcomes of the study up to day 28

\begin{tabular}{|c|c|c|c|}
\hline Variable & Melatonin group (20 patients) & Placebo group (20 patients) & $p$ value \\
\hline 28-day mortality, no. (\%) & $5(25.0)$ & $8(40.0)$ & 0.51 \\
\hline Number of patients needed mechanical ventilation up to day 28 , no. (\%) & $9(45.0)$ & $13(65.0)$ & 0.33 \\
\hline \multicolumn{4}{|l|}{ Ventilator-free days to day 28} \\
\hline Mean \pm SD & $16.90 \pm 9.24$ & $10.00 \pm 10.94$ & \multirow[t]{2}{*}{$\mathbf{0 . 0 3 5}$} \\
\hline Median (IQR) & $18(26.50-11.50)$ & $7.00(17.00-0.00)$ & \\
\hline Number of patients who needed vasopressor up to day 28, no. $(\%)$ & $20(100)$ & $20(100)$ & 1.00 \\
\hline \multicolumn{4}{|l|}{ Vasopressor-free days to day 28} \\
\hline Mean \pm SD & $12.75 \pm 7.43$ & $10.15 \pm 6.12$ & \multirow[t]{2}{*}{0.046} \\
\hline Median (IQR) & $11.50(17.25-7.50)$ & $8.50(12.75-6.25)$ & \\
\hline \multicolumn{4}{|l|}{ Change in mean vasopressor dose from enrollment to day $5(\mathrm{mcg} / \mathrm{min})$} \\
\hline Mean \pm SD & $-6.21 \pm 5.12$ & $-3.20 \pm 3.95$ & \multirow[t]{2}{*}{0.045} \\
\hline Median (IQR) & $-5.00(-2.75-(-9.25))$ & $-5.00(0.00-(-5.00))$ & \\
\hline \multicolumn{4}{|l|}{ Change in mean SOFA score from enrollment to day 5} \\
\hline Mean \pm SD & $-4.05 \pm 4.75$ & $-2.25 \pm 4.87$ & \multirow[t]{2}{*}{0.24} \\
\hline Median (IQR) & $-5.00(-0.25-(-6.75))$ & $-3.00(0.75-(-6.75))$ & \\
\hline Number of patients with SOFA scores less than 6 at day 28 , no. (\%) & $12(60.0)$ & $7(35.0)$ & 0.21 \\
\hline Number of patients needed renal replacement therapy to day 28, no. (\%) & $3(15.0)$ & $5(25.0)$ & 0.69 \\
\hline \multicolumn{4}{|l|}{ Length of ICU stay, days } \\
\hline Mean \pm SD & $15.85 \pm 5.03$ & $21.10 \pm 10.12$ & \multirow[t]{2}{*}{0.045} \\
\hline Median (IQR) & $16.00(19.00-12.50)$ & $21.00(28.00-14.00)$ & \\
\hline \multicolumn{4}{|l|}{ Length of hospital stay, days } \\
\hline Mean \pm SD & $18.05 \pm 5.14$ & $25.85 \pm 7.13$ & \multirow[t]{2}{*}{$\mathbf{0 . 0 3}$} \\
\hline Median (IQR) & $19(21-15)$ & $23(36-15)$ & \\
\hline
\end{tabular}

$S D$ standard deviation, IQR interquartile range, SOFA Sequential Organ Failure Assessment, ICU intensive care unit. The significant $p$ value is shown in bold type

administered melatonin might also be a promising agent in modulating the oxidative stress and inflammatory state seen in sepsis. From a pathophysiology viewpoint, growing evidence indicates that the circadian rhythm of melatonin secretion has been disrupted during severe sepsis [16, 34-36]. It is reported that there is an association between the low melatonin levels and impairment of immune system function, increased severity of infection, and worsening clinical outcomes in the sepsis setting $[18,37]$. Melatonin, as a major intracellular antioxidant, through multiple potential actions, including direct free radical scavenging, stimulation of antioxidative enzymes such as glutathione reductase, superoxide dismutase, and glutathione peroxidase, enhancing the mitochondrial oxidative phosphorylation efficiency and decreasing electron leakage and as a result, reducing free radical generation, and enhancing the efficacy of other antioxidants can exert protective effects against oxidative stressinduced damage to molecules, cells, and tissues [38]. It is found that melatonin's metabolites also possess antioxidant activity and can deactivate a wide variety of ROS radicals [39]. It seems that decreased serum melatonin levels during severe septic at least impart related to its rapid utilization as an antioxidant [40]. Due to its high lipophilicity and small size, as well as the presence of the active melatonin transport in the mitochondrial membrane, melatonin is able to enter and accumulate within mitochondria in a dose-dependent manner [41, 42]. This evidence suggests that melatonin as an antioxidant that acts in mitochondria can exert protective effects against mitochondrial damage resulting from oxidative stress [43]. Interestingly enough, in a study in an acute model of sepsis, melatonin demonstrated similar effects to the synthetic mitochondrial antioxidants like MitoE and MitoQ regarding protection against pro-inflammatory cytokine responses, oxidative stress, and mitochondrial dysfunction in the setting of sepsis [44]. While it is found that an increase in the production and activity of nitric oxide (NO) by the inducible nitric oxide synthase (iNOS) has a crucial role in mitochondrial dysfunction in septic setting [45], research in animal models of sepsis shows that melatonin has inhibitory effects on induction of iNOS and reduces NO production in septic mice [22, 37, 46, 47].

Beyond this, there is a wealth of evidence that melatonin possesses high anti-inflammatory properties. It has been found that melatonin modulates inflammatory processes without impairing the physiologic inflammatory responses [48]. However, at present, the exact mechanisms underlying the anti-inflammatory effects of melatonin are still not fully elucidated. Given the close connections between 
inflammation and oxidative stress and the overproduction of ROS during oxidative stress contributes to the development and perpetuation of the inflammatory process [4], it is speculated that the main anti-inflammatory action of melatonin could be mediated through its antioxidant activities [49]. Further, it is found that melatonin in a dose-dependent fashion through modulation of nuclear factor kappa-B (NF- $\kappa \mathrm{B})$ and Nod-like receptor pyrin containing 3 (NLRP3) signaling pathway that their interaction is responsible for activating pro-inflammatory responses during sepsis can regulate the balance of anti- and pro-inflammatory cytokines in this inflammatory-mediated pathological condition [50]. In this context, a number of experimental studies in the models of sepsis demonstrated that melatonin, through inhibition of the NF- $\mathrm{KB} / \mathrm{NLRP} 3$ pathway activation, could regulate the pro-inflammatory mediators' production and restore the mitochondrial homeostasis [47, 51-56].

Apart from the above mechanisms, recently, preliminary evidence raises regarding the medical application of melatonin, as a potential antimicrobial agent, in the prevention and treatment of various bacterial and viral infections [57]. In this context, some ex vivo and in vivo studies reported the antimicrobial activities of melatonin against both Gramnegative and Gram-positive pathogenic bacteria, even problematic bacteria, such as carbapenem-resistant Pseudomonas aeruginosa, Acinetobacter baumannii, and Staphylococcus aureus, causing antibiotic-resistant nosocomial infections [58-60]. The impact of melatonin in restricting bacterial growth is likely, in part, to be attributed to its ability to bind metals (e.g., $\mathrm{Fe}+3$ ) and reducing the availability of intracellular substrates [58].

According to this evidence, it appears that the potential benefits of melatonin in the septic setting are mediated through a broad spectrum of actions. To date, in a number of animal models of sepsis and septic shock, the potential effectiveness of melatonin has been widely investigated. Results of these studies strongly support the hypothesis that melatonin and melatonin receptor agonists can limit sepsisinduced organ dysfunction, and patients with sepsis might benefit from these natural agents $[19,61-65]$. It is found that exogenous melatonin administration in the animal model of sepsis could be able to restore the normal homeostasis in all organs [47, 51]. Further, it has been shown that the melatonin's protective mechanisms against sepsis are totally antagonized by a co-treatment with the melatonin receptor antagonist like luzindole. This evidence indicates that the melatonin's protective actions on septic organ injuries might be mediated via its membrane receptors (MT1/MT2 receptors) [62]. According to the experimental evidence, time, dose, and length of melatonin administration also need to be considered in the treatment of septic, as it is speculated that melatonin therapy at high dosage in the early phase of septic and for a short duration could exert benefits in septic patients
$[66,67]$. Due to circulatory dysfunction, drug absorption is compromised in septic patients [68]. On the other hand, due to a substantial first-pass metabolism, melatonin has a low oral bioavailability, with a range from 3 to $33 \%$ [69, 70], so this evidence indicates that a large oral dosage of melatonin (around $50 \mathrm{mg}$ of melatonin) is likely needed to exerts its dose-dependent antioxidant and anti-inflammatory effects in the immunopathological conditions, like sepsis [71]. Concerning melatonin dosing, it has also been recognized that melatonin administration in multiple daily doses also might be important to optimize melatonin's anti-inflammatory and antioxidant efficacy in pathological conditions $[72,73]$. Further, currently in the animal model of sepsis, it has been found that delivering melatonin with specific drug delivery systems can improve its antioxidant effects against oxidative stress-induced damage in the septic setting [21].

However, despite this strong scientific evidence, to date, relatively little clinical finding exists concerning melatonin efficacy as a therapeutic approach in the management of septic shock. To date, some limited clinical studies have been conducted regarding the effectiveness of melatonin as adjuvant therapy in neonates with sepsis [74-77]. A recent meta-analysis on these studies with a total of 120 study subjects concluded that adjunctive therapy with melatonin during the initial $24 \mathrm{~h}$ post-diagnosis of sepsis could be able to decrease serum levels of C-reactive protein as an inflammatory indicator and improve the clinical outcomes in the neonatal sepsis [78]. In a recent controlled clinical trial, Aisa-Alvarez and co-workers investigated the potential efficacy of several antioxidants, including vitamin $\mathrm{C}$ (group 1: at a dosage of $1000 \mathrm{mg}$ every $6 \mathrm{~h}$ ), vitamin $\mathrm{E}$ (group 2: at a dosage of $400 \mathrm{IU}$ every $8 \mathrm{~h}$ ), n-acetylcysteine (NAC) (group 3: at a dosage of $600 \mathrm{mg}$ every $12 \mathrm{~h}$ ), and melatonin (group 4: at a dosage of $50 \mathrm{mg}$ once daily) as adjuvant therapy to standard-of-care treatment for five consecutive days in comparison to only standard-of-care treatment (group 5) in adult patients with septic shock presented with multiple organ dysfunction [79]. Results of the study demonstrated that each of these antioxidants, particularly vitamin $\mathrm{C}$ and melatonin, has beneficial effects on decreasing the severity of organ dysfunction assessed based on the SOFA score. Further, a significant decrease in the plasma level of oxidative stress and proinflammatory biomarkers such as C-reactive protein (CRP), procalcitonin, and lipid-peroxidation have been observed in the patients who received melatonin [79]. Consistent with these findings, our study also provided clinical evidence regarding melatonin's beneficial effects on adult septic shock. Besides these findings, previously, results of a randomized, doubleblind placebo-controlled study showed that melatonin supplementation at a dosage of $10 \mathrm{mg}$ at night could improve nocturnal sleep in critically ill patients receiving mechanical ventilation [80]. Castroviejo et al., in their recent clinical 
trial (EudraCT: 2008-006,782-83) that its results still do not publish, observed that intravenous administration of $60 \mathrm{mg} /$ day melatonin could significantly reduce mortality in septic patients [81]. During the current global pandemic, scientific evidence is also rising regarding the potential benefits of melatonin as adjuvant therapy in preventing and treating coronavirus disease 2019 (COVID-19) infection [82]. Considering the critical role of excessive inflammatory and oxidative stress responses in the pathogenesis of pulmonary and systemic damage in severe COVID-19, it is not surprising noting that the preliminary clinical findings were promising on melatonin's utility in patients with severe COVID-19 infections [72, 83].

While there is limited clinical evidence in the critically ill patient population concerning melatonin's safety, many experimental and clinical research works have reported melatonin as a safe agent, without any serious or clinical important adverse events even at supra-physiologic doses [84]. In this context, recently, in a phase I dose-escalation research on healthy cases, melatonin at high single oral doses up to $100 \mathrm{mg}$ was well tolerated, and no clinically significant changes to any biochemical or physiological measures were observed [71].

Thus, the light of the current data in the literature suggests that adjunctive therapy with melatonin might be safe and effective in improving clinical outcomes of critically ill patients, such as septic patients. However, these findings require confirmation. Our study could give us some clinical evidence regarding efficacy of melatonin in septic shock. Further, at present, there are a number of ongoing clinical trials on evaluating the dose, safety, and efficacy of melatonin in treating severe sepsis and septic shock that; the obtained results from these trials can help to clarify the potential usefulness of melatonin in sepsis treatment.

Although the results of our study are promising, due to the preliminary nature of our work, it suffers from some limitations that should be mentioned. Small sample size, the short period of follow-up, and a single-center investigation are some of the major limitations of the trial. Since the sample size of our study was relatively small, it was not powered to assess the benefits of melatonin therapy on the mortality rate of septic patients. Thus, despite achieving promising results in some clinical outcomes, we did not conclude whether treatment with melatonin could reduce mortality in these patients or not. Additionally, because of structural constraints, the melatonin serum levels were not measured before and after the treatment. Further, we did not assess the changes in the serum levels of inflammatory and oxidative stress biomarkers in the study patients. So, it did not allow identifying the regulatory effects of melatonin on immunoinflammatory mediators as its possible mechanism of action against sepsis. Therefore, additional research with a higher sample size and a more length follow-up period is required to address these issues in-depth.

\section{Conclusion}

In summary, data from the present study provided preliminary clinical evidence that administering a high dosage of melatonin ( $50 \mathrm{mg} /$ day for 5 days) early after the onset of septic shock as an add-on therapy to standard treatment care might be beneficial in improving some clinical outcomes in septic shock patients. Thus, the scientific evidence on the potential utility of melatonin in treating septic shock was supported by our findings. Conducting future clinical research could be valuable to replicate and expand these findings.

Abbreviations SBP: Systolic blood pressure; ROS: Reactive oxygen species; ICU: Intensive care unit; SD: Standard deviation; SOFA: Sequential Organ Failure Assessment; ScvO2: Central venous oxygen saturation; APACHE: Acute Physiology and Chronic Health Evaluation; ITT: Intentto-treat; LOCF: Last observation carried forward; IQR: Interquartile range; ANOVA: Analysis of variance; NO: Nitric oxide; iNOS: Inducible nitric oxide synthase, NF-кB, nuclear factor kappa-B; NLRP3: Nod-like receptor pyrin containing 3; NAC: N-acetylcysteine; CRP: C-reactive protein; COVID-19: Coronavirus disease 2019

Acknowledgements We thank all the patients and medical staff of Besat Hospital that have contributed to this study.

Author contribution Conceptualization: Mehrpooya and Shokoohmand. Methodology: Taher, Mehrpooya, and Shokoohmand. Data acquisition: Taher, abdoli, and Shokoohmand. Statistical analysis: Mohammadi. Writing - original draft preparation: Shokoohmand. Writing-review and editing: Mehrpooya and Taher. All authors contributed to the interpretation of the results and read and approved the final manuscript.

Funding This research was supported by funding from the vicechancellor for research and technology, Hamadan University of Medical Sciences, Hamadan, Iran (No: 9808286519). This grant was not assigned to the manuscript writing, editing, and publication fee.

Availability of data and material The datasets used and analyzed during the current study are available from the corresponding author on reasonable request up to 2 years after publication.

Code availability Not applicable.

\section{Declarations}

Ethics approval and consent to participate The trial protocol was according to the Declaration of Helsinki as revised in 1989, and the study protocol was approved by the research and ethics committee at Hamadan University of Medical Sciences (IR.UMSHA. REC.1398.341).

Consent for publication All authors have given consent for publication.

Competing interests The authors declare no competing interests. 


\section{References}

1. Physicians ACoC (1992) Society of Critical Care Medicine Consensus Conference Committee: American College of Chest Physicians/Society of Critical Care Medicine Consensus Conference: definitions for sepsis and organ failure and guidelines for the use of innovative therapies in sepsis. Crit Care Med 20:864-874

2. Singer M, Deutschman CS, Seymour CW et al (2016) The third international consensus definitions for sepsis and septic shock (Sepsis-3). JAMA 315(8):801-810

3. Galley HF (2011) Oxidative stress and mitochondrial dysfunction in sepsis. Br J Anaesth 107(1):57-64

4. Lugrin J, Rosenblatt-Velin N, Parapanov R, Liaudet L (2014) The role of oxidative stress during inflammatory processes. Biol Chem 395(2):203-230

5. Nagar H, Piao S, Kim C-S (2018) Role of mitochondrial oxidative stress in sepsis. Acute and critical care 33(2):65

6. Duran-Bedolla J, de Oca-Sandoval MAM, Saldaña-Navor V et al (2014) Sepsis, mitochondrial failure and multiple organ dysfunction. Clin Invest Med E58-E69

7. Mantzarlis K, Tsolaki V, Zakynthinos E (2017) Role of oxidative stress and mitochondrial dysfunction in sepsis and potential therapies. Oxidative medicine and cellular longevity 2017

8. Pandi-Perumal SR, Trakht I, Srinivasan V et al (2008) Physiological effects of melatonin: role of melatonin receptors and signal transduction pathways. Prog Neurobiol 85(3):335-353

9. Nowak J, Zawilska J (1998) Melatonin and its physiological and therapeutic properties. Pharm World Sci 20(1):18-27

10. Mahi-Birjand M, Yaghoubi S, Abdollahpour-Alitappeh $\mathrm{M}$ et al (2020) Protective effects of pharmacological agents against aminoglycoside-induced nephrotoxicity: a systematic review. Expert Opin Drug Saf 19(2):167-186

11. Maharaj DS, Glass BD, Daya S (2007) Melatonin: new places in therapy. Biosci Rep 27(6):299-320

12. Ferlazzo N, Andolina G, Cannata A et al (2020) Is Melatonin the Cornucopia of the 21st Century? Antioxidants 9(11):1088

13. Lin G-J, Huang S-H, Chen S-J et al (2013) Modulation by melatonin of the pathogenesis of inflammatory autoimmune diseases. Int J Mol Sci 14(6):11742-11766

14. Srinivasan V, Pandi-Perumal SR, Spence DW et al (2010) Melatonin in septic shock: some recent concepts. J Crit Care 25(4):656.e1-656.e6

15. Acuña-Castroviejo D, Rahim I, Acuña-Fernández C et al (2017) Melatonin, clock genes and mitochondria in sepsis. Cell Mol Life Sci 74(21):3965-3987

16. Mundigler G, Delle-Karth G, Koreny M et al (2002) Impaired circadian rhythm of melatonin secretion in sedated critically ill patients with severe sepsis. Crit Care Med 30(3):536-540

17. Frisk U, Olsson J, Nylén P, Hahn RG (2004) Low melatonin excretion during mechanical ventilation in the intensive care unit. Clin Sci 107(1):47-53

18. Perras B, Kurowski V, Dodt C (2006) Nocturnal melatonin concentration is correlated with illness severity in patients with septic disease. Intensive Care Med 32(4):624-625

19. Carrillo-Vico A, Lardone PJ, Naji L et al (2005) Beneficial pleiotropic actions of melatonin in an experimental model of septic shock in mice: regulation of pro-/anti-inflammatory cytokine network, protection against oxidative damage and anti-apoptotic effects. J Pineal Res 39(4):400-408

20. Ortiz F, García JA, Acuña-Castroviejo D et al (2014) The beneficial effects of melatonin against heart mitochondrial impairment during sepsis: Inhibition of $\mathrm{i}$ NOS and preservation of $\mathrm{n}$ NOS. J Pineal Res 56(1):71-81

21. Li Volti G, Musumeci T, Pignatello R et al (2012) Antioxidant potential of different melatonin-loaded nanomedicines in an experimental model of sepsis. Exp Biol Med 237(6):670-677
22. Wu JY, Tsou MY, Chen TH et al (2008) Therapeutic effects of melatonin on peritonitis-induced septic shock with multiple organ dysfunction syndrome in rats. J Pineal Res 45(1):106-116

23. Zabet MH, Mohammadi M, Ramezani M, Khalili H (2016) Effect of high-dose ascorbic acid on vasopressor's requirement in septic shock. Journal of research in pharmacy practice 5(2): 94

24. Vincent J-L, De Mendonça A, Cantraine F et al (1998) Use of the SOFA score to assess the incidence of organ dysfunction/failure in intensive care units: results of a multicenter, prospective study. Crit Care Med 26(11):1793-1800

25. Rhodes A, Evans LE, Alhazzani W et al (2017) Surviving sepsis campaign: international guidelines for management of sepsis and septic shock: 2016. Intensive Care Med 43(3):304-377

26. Patergnani S, Bouhamida E, Leo S et al (2021) Mitochondrial Oxidative Stress and "Mito-Inflammation": Actors in the Diseases. Biomedicines 9(2):216

27. Zhang H, Feng Y-W, Yao Y-M (2018) Potential therapy strategy: targeting mitochondrial dysfunction in sepsis. Mil Med Res 5(1):1-11

28. Macdonald J, Galley HF, Webster NR (2003) Oxidative stress and gene expression in sepsis. Br J Anaesth 90(2):221-232

29. Schulte W, Bernhagen J, Bucala R (2013) Cytokines in sepsis: potent immunoregulators and potential therapeutic targets-an updated view. Mediat Inflamm 2013

30. Chertoff $\mathbf{J}(2018) \mathrm{N}$-acetylcysteine's role in sepsis and potential benefit in patients with microcirculatory derangements. J Intensive Care Med 33(2):87-96

31. Marik PE, Khangoora V, Rivera R et al (2017) Hydrocortisone, vitamin $\mathrm{C}$, and thiamine for the treatment of severe sepsis and septic shock: a retrospective before-after study. Chest 151(6):1229-1238

32. Rank N, Michel C, Haertel C et al (2000) N-acetylcysteine increases liver blood flow and improves liver function in septic shock patients: results of a prospective, randomized, double-blind study. Crit Care Med 28(12):3799-3807

33. Macarthur H, Couri DM, Wilken GH et al (2003) Modulation of serum cytokine levels by a novel superoxide dismutase mimetic, M40401, in an Escherichia coli model of septic shock: correlation with preserved circulating catecholamines. Crit Care Med 31(1):237-245

34. Verceles AC, Silhan L, Terrin M et al (2012) Circadian rhythm disruption in severe sepsis: the effect of ambient light on urinary 6-sulfatoxymelatonin secretion. Intensive Care Med 38(5):804-810

35. Olofsson K, Alling C, Lundberg D, Malmros C (2004) Abolished circadian rhythm of melatonin secretion in sedated and artificially ventilated intensive care patients. Acta Anaesthesiol Scand 48(6):679-684

36. Li C-X, Liang D-D, Xie G-H et al (2013) Altered melatonin secretion and circadian gene expression with increased proinflammatory cytokine expression in early-stage sepsis patients. Mol Med Rep 7(4):1117-1122

37. Escames G, León J, Macías M et al (2003) Melatonin counteracts lipopolysaccharide-induced expression and activity of mitochondrial nitric oxide synthase in rats. FASEB J 17(8):1-22

38. Reiter RJ, Tan D-X, Mayo JC et al (2003) Melatonin as an antioxidant: biochemical mechanisms and pathophysiological implications in humans. Acta Biochim Pol 50(4):1129-1146

39. Galano A, Tan DX, Reiter RJ (2013) On the free radical scavenging activities of melatonin's metabolites, AFMK and AMK. J Pineal Res 54(3):245-257

40. Jiang-Shieh $\mathrm{YF}, \mathrm{Wu} \mathrm{CH}$, Chien $\mathrm{HF}$ et al (2005) Reactive changes of interstitial glia and pinealocytes in the rat pineal gland challenged with cell wall components from gram-positive and-negative bacteria. J Pineal Res 38(1):17-26

41. Venegas C, García JA, Escames G et al (2012) Extrapineal melatonin: analysis of its subcellular distribution and daily fluctuations. J Pineal Res 52(2):217-227 
42. Hevia D, González-Menéndez P, Quiros-González I et al (2015) Melatonin uptake through glucose transporters: a new target for melatonin inhibition of cancer. J Pineal Res 58(2):234-250

43. Tan D-X, Manchester LC, Qin L, Reiter RJ (2016) Melatonin: a mitochondrial targeting molecule involving mitochondrial protection and dynamics. Int J Mol Sci 17(12):2124

44. Lowes D, Webster N, Murphy M, Galley H (2013) Antioxidants that protect mitochondria reduce interleukin- 6 and oxidative stress, improve mitochondrial function, and reduce biochemical markers of organ dysfunction in a rat model of acute sepsis. Br J Anaesth 110(3):472-480

45. Boveris A, Alvarez S, Navarro A (2002) The role of mitochondrial nitric oxide synthase in inflammation and septic shock. Free Radical Biol Med 33(9):1186-1193

46. López LC, Escames G, Tapias V et al (2006) Identification of an inducible nitric oxide synthase in diaphragm mitochondria from septic mice: its relation with mitochondrial dysfunction and prevention by melatonin. Int J Biochem Cell Biol 38(2):267-278

47. Escames G, López LC, Ortiz F et al (2007) Attenuation of cardiac mitochondrial dysfunction by melatonin in septic mice. FEBS J 274(8):2135-2147

48. Zarezadeh M, Khorshidi M, Emami M et al (2019) Melatonin supplementation and pro-inflammatory mediators: a systematic review and meta-analysis of clinical trials. Eur J Nutr 1-11

49. Reiter RJ, Mayo JC, Tan DX et al (2016) Melatonin as an antioxidant: under promises but over delivers. J Pineal Res 61(3):253-278

50. Mauriz JL, Collado PS, Veneroso C et al (2013) A review of the molecular aspects of melatonin's anti-inflammatory actions: recent insights and new perspectives. J Pineal Res 54(1):1-14

51. Escames G, Acuña-Castroviejo D, López LC (2006) Tan Dx, Maldonado MD, Sánchez-Hidalgo M, León J, Reiter RJ: Pharmacological utility of melatonin in the treatment of septic shock: experimental and clinical evidence. J Pharm Pharmacol 58(9):1153-1165

52. Rahim I, Djerdjouri B, Sayed RK et al (2017) Melatonin administration to wild-type mice and nontreated NLRP 3 mutant mice share similar inhibition of the inflammatory response during sepsis. J Pineal Res 63(1):e12410

53. García JA, Volt H, Venegas $C$ et al (2015) Disruption of the $\mathrm{NF}-\kappa \mathrm{B} / \mathrm{NLRP} 3$ connection by melatonin requires retinoid-related orphan receptor- $\alpha$ and blocks the septic response in mice. FASEB J 29(9):3863-3875

54. An R, Zhao L, Xi C et al (2016) Melatonin attenuates sepsisinduced cardiac dysfunction via a PI3K/Akt-dependent mechanism. Basic Res Cardiol 111(1):8

55. Şener G, Toklu H, Kapucu C et al (2005) Melatonin protects against oxidative organ injury in a rat model of sepsis. Surg Today 35(1):52-59

56. Volt H, García JA, Doerrier C et al (2016) Same molecule but different expression: aging and sepsis trigger NLRP3 inflammasome activation, a target of melatonin. J Pineal Res 60(2):193-205

57. Hu W, Deng C, Ma Z et al (2017) Utilizing melatonin to combat bacterial infections and septic injury. Br J Pharmacol 174(9):754-768

58. Tekbas OF, Ogur R, Korkmaz A et al (2008) Melatonin as an antibiotic: new insights into the actions of this ubiquitous molecule. $\mathrm{J}$ Pineal Res 44(2):222-226

59. Celinski K, Konturek P, Konturek S et al (2011) Effects of melatonin and tryptophan on healing of gastric and duodenal ulcers with Helicobacter pylori infection in humans. J Physiol Pharmacol 62(5):521

60. Wu UI, Mai FD, Sheu JN et al (2011) Melatonin inhibits microglial activation, reduces pro-inflammatory cytokine levels, and rescues hippocampal neurons of adult rats with acute Klebsiella pneumoniae meningitis. J Pineal Res 50(2):159-170
61. Chen HH, Lin KC, Wallace CG et al (2014) Additional benefit of combined therapy with melatonin and apoptotic adipose-derived mesenchymal stem cell against sepsis-induced kidney injury. J Pineal Res 57(1):16-32

62. Fink T, Glas M, Wolf A et al (2014) Melatonin receptors mediate improvements of survival in a model of polymicrobial sepsis. Crit Care Med 42(1):e22-e31

63. Shang Y, Xu S-P, Wu Y et al (2009) Melatonin reduces acute lung injury in endotoxemic rats. Chin Med J 122(12):1388-1393

64. Sewerynek E, Melchiorri D, Reiter RJ et al (1995) Lipopolysaccharide-induced hepatotoxicity is inhibited by the antioxidant melatonin. Eur J Pharmacol Environ Toxicol Pharm 293(4):327-334

65. Wu CC, Chiao CW, Hsiao G et al (2001) Melatonin prevents endotoxin-induced circulatory failure in rats. J Pineal Res 30(3):147-156

66. Annane D, Bellissant E, Cavaillon J-M (2005) Septic shock. The Lancet 365(9453):63-78

67. Wichmann MW, Haisken JM, Ayala A, Chaudry IH (1996) Melatonin administration following hemorrhagic shock decreases mortality from subsequent septic challenge. J Surg Res 65(2):109-114

68. De Paepe P, Belpaire FM, Buylaert WA (2002) Pharmacokinetic and pharmacodynamic considerations when treating patients with sepsis and septic shock. Clin Pharmacokinet 41(14):1135-1151

69. DeMuro RL, Nafziger AN, Blask DE et al (2000) The absolute bioavailability of oral melatonin. J Clin Pharmacol 40(7):781-784

70. Fourtillan J, Brisson A, Gobin P et al (2000) Bioavailability of melatonin in humans after day-time administration of D7 melatonin. Biopharm Drug Dispos 21(1):15-22

71. Galley HF, Lowes DA, Allen L et al (2014) Melatonin as a potential therapy for sepsis: a phase I dose escalation study and an ex vivo whole blood model under conditions of sepsis. J Pineal Res 56(4):427-438

72. Castillo RR, Quizon GRA, Juco MJM et al (2020) Melatonin as adjuvant treatment for coronavirus disease 2019 pneumonia patients requiring hospitalization (MAC-19 PRO): a case series. Melatonin Res 3(3):297-310

73. DiNicolantonio JJ, McCarty M, Barroso-Aranda J (2021) Melatonin may decrease risk for and aid treatment of COVID-19 and other RNA viral infections. In.: Archives of Disease in childhood

74. Gitto E, Karbownik M, Reiter RJ et al (2001) Effects of melatonin treatment in septic newborns. Pediatr Res 50(6):756-760

75. El Frargy M, El-Sharkawy H, Attia G (2015) Use of melatonin as an adjuvant therapy in neonatal sepsis. J Neonatal-Perinatal Med 8(3):227-232

76. El-Gendy FM, El-Hawy MA, Hassan MG (2018) Beneficial effect of melatonin in the treatment of neonatal sepsis. J Matern Fetal Neonatal Med 31(17):2299-2303

77. Gitto E, Reiter RJ, Cordaro SP et al (2004) Oxidative and inflammatory parameters in respiratory distress syndrome of preterm newborns: beneficial effects of melatonin. Am J Perinatol 21(04):209-216

78. Henderson R, Kim S, Lee E (2018) Use of melatonin as adjunctive therapy in neonatal sepsis: a systematic review and meta-analysis. Complement Ther Med 39:131-136

79. Aisa-Alvarez A, Soto ME, Guarner-Lans V et al (2020) Usefulness of antioxidants as adjuvant therapy for septic shock: A randomized clinical trial. Medicina 56(11):619

80. Bourne RS, Mills GH, Minelli C (2008) Melatonin therapy to improve nocturnal sleep in critically ill patients: encouraging results from a small randomised controlled trial. Crit Care 12(2):1-9

81. Acuña-Castroviejo D, Escames G, Figueira JC et al (2020) Clinical trial to test the efficacy of melatonin in COVID-19. J Pineal Res 69(3):e12683 
82. Shneider A, Kudriavtsev A, Vakhrusheva A (2020) Can melatonin reduce the severity of COVID-19 pandemic? Int Rev Immunol 39(4):153-162

83. Ramlall V, Zucker J, Tatonetti N (2020) Melatonin is significantly associated with survival of intubated COVID-19 patients. medRxiv
84. Andersen LPH, Gögenur I, Rosenberg J, Reiter RJ (2016) The safety of melatonin in humans. Clin Drug Investig 36(3):169-175

Publisher's Note Springer Nature remains neutral with regard to jurisdictional claims in published maps and institutional affiliations. 